\title{
Prognostic Significance of P16, EZH2, FOXJ1, and Tenascin Expression in Pediatric Ependymoma
}

\author{
Sadeq Wasil Ali Al-Dandanª, b, , Syed Nizam Hussain ${ }^{\mathrm{c}}$, Musa Al-Harbic
}

\begin{abstract}
Background: The prognosis of pediatric ependymomas remains poor, with treatment being predominantly based on surgery with or without radiotherapy. The lack of robust therapeutic molecular markers for clinical use has hampered attempts to improve survival from ependymomas. This study aimed to assess the prognostic significance of p16, enhancer of zeste homolog 2 (EZH2), forkhead box protein J 1 (FOXJ1), and tenascin in pediatric ependymomas by evaluating their immunohistochemical expression and comparing them with clinical outcomes.
\end{abstract}

Methods: A total of 22 children ( $<14$ years) with ependymoma were retrospectively analyzed for the expression of p16, EZH2, FOXJ1, and tenascin by immunohistochemical staining. Kaplan-Meier analysis was used to evaluate the association between immunohistochemical marker expression and patient survival.

Results: Higher expression of p16, EZH2, FOXJ1, and tenascin was observed in $59.0 \%, 36.3 \%, 40.9 \%$, and $68.1 \%$ of the ependymoma samples, respectively. Patients with p16-positive and p16-negative tumors had an overall median survival time of 5.38 and 3.38 years and an overall cumulative survival rate of $44.5 \%$ and $36.5 \%$, respectively. P16 negativity was significantly associated with poorer outcome $(\mathrm{P}=0.009)$. No relationship was observed between EZH2, FOXJ1, and tenascin expression and overall survival ( $\mathrm{P}=0.904,0.844$, and 0.646 , respectively).

Conclusions: Loss of p16 expression was associated with poor prognosis and may be used for risk stratification. Limitations of the present study include its small sample size and variable sensitivity of different antibody clones and detection methods.

Keywords: Ependymoma; Immunohistochemistry; Prognosis; Pediatric brain tumor

Manuscript submitted June 8, 2020, accepted June 15, 2020

Published online July 30, 2020

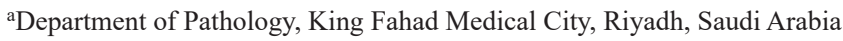
bepartment of Pathology, Maternity and Children Hospital Al-Ahsa, Al-Ahsa, Saudi Arabia

'Department of Pediatric Hemato-Oncology, Comprehensive Cancer Center, King Fahad Medical City, Riyadh, Saudi Arabia

${ }^{\mathrm{d} C}$ Corresponding Author: Sadeq Wasil Ali Al-Dandan, Department of Pathology, Maternity and Children Hospital Al-Ahsa, Al-Ahsa, Saudi Arabia.

Email: sadeqdan@gmail.com

doi: https://doi.org/10.14740/ijcp385

\section{Introduction}

Ependymomas are neuroepithelial tumors that arise throughout the neuraxis usually in association with ventricular cavities or the central spinal canal. Ependymomas account for approximately $10 \%$ of all brain tumors in the pediatric population [1] and are the third most common intracranial brain tumor among children (after medulloblastomas and astrocytomas). They arise from radial glia cells from the corresponding central nervous system regions [2]. Posterior fossa ependymomas comprise two-thirds of all cases, while supratentorial ependymomas represent less than a third, with the rest residing in the spine [3].

Treatment has predominantly included surgery with or without radiotherapy but not chemotherapy considering that ependymomas are chemo-resistant [4]. The prognosis of pediatric ependymomas remains poor with a 5-year progressionfree survival of between $30 \%$ and $69.1 \%$ [5]. Increasing age, spinal location, and maximal surgical resection have been the best clinical prognostic factors associated with improved survival [5]. Attempts to improve ependymomas survival have been hampered by several factors, including the rarity of the disease, lack of effective chemotherapy, difficulty in histopathologic grading, and paucity of robust therapeutic molecular markers for clinical use $[6,7]$.

This study aimed to assess the prognostic significance of p16, enhancer of zeste homolog 2 (EZH2), forkhead box protein J 1 (FOXJ1), and tenascin in pediatric ependymomas by evaluating their immunohistochemical expression and comparing them with clinical outcomes.

\section{Materials and Methods}

A retrospective chart review of medical records was performed for children (14 years old and under) diagnosed with ependymoma between 2004 and 2016 at King Fahad Medical City, Riyadh, Saudi Arabia. Institutional Review Board (IRB) approval was obtained before conducting the study. This study was conducted in compliance with the ethical standards of the responsible institution on human subjects as well as with the Helsinki Declaration. Inclusion criteria included a histopathologic diagnosis of ependymoma, age $\leq 14$ years, availability of clinical data, and paraffin-embedded tissue blocks. Exclusion criteria included the absence of tissue blocks and insufficient 


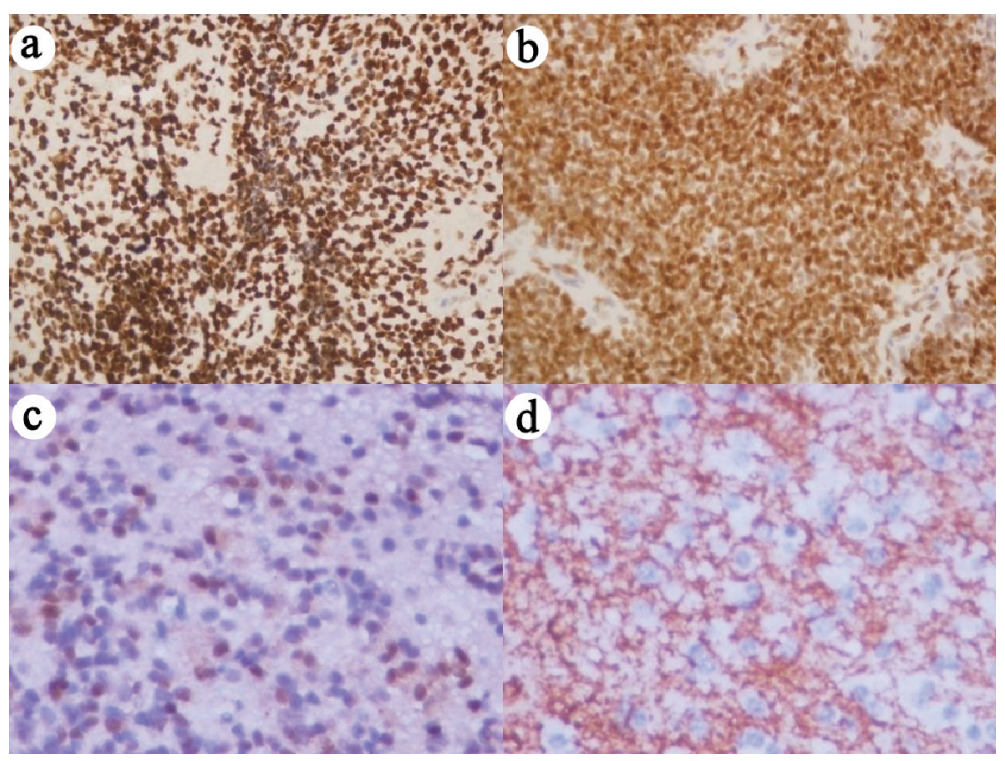

Figure 1. Immunohistochemical staining with high expression for (a) p16, (b) EZH2, (c) FOXJ1, and (d) Tenascin ( 400 magnification). EZH2: enhancer of zeste homolog 2; FOXJ1: forkhead box protein J 1.

clinical data. Clinical data obtained included age, tumor location, mode of treatment, tumor recurrence, and patient survival.

Histologic slides were thoroughly reviewed for diagnostic confirmation according to the World Health Organization (WHO) grading system. The selected paraffin-embedded tissue blocks were cut into 5 - $\mu \mathrm{m}$ sections and mounted on positively charged microscopic slides. A polymer-based detection system was used for immunohistochemical testing. The slides were then stained with p16 (CINtec Histology Kit, E6H4; Roche mtm Laboratories AG, Basel, Switzerland), EZH2 (SP129, Roche mtm Laboratories AG), FOXJ1 (clone 3 - 19 (05-837); EMD Millipore, MA, USA), and tenascin (AB19013; EMD Millipore). Both positive and negative controls were performed.

Scoring of the immunohistochemical staining (IHC) was performed by a board-certified neuropathologist who was blinded to clinical data and survival. A semi-quantitative approach that combined the intensity of the immunohistochemical reaction and the percentage of positive cells was used. The intensity of the staining was graded from 0 to 3 ( $0=$ no staining, 1 = weak staining, $2=$ moderate staining, and $3=$ strong staining). The percentage of cells with staining was graded from 0 to $4(1=1-25 \%, 2=25-50 \%, 3=50-75 \%$, and $4=$ $75-100 \%$ ). The final score was obtained by multiplying the intensity by the percentage. Total scores of $\leq 8$ and $9-12$ were considered IHC-low and IHC-high, respectively.

Kaplan-Meier analysis was used to estimate survival differences, while the log-rank test (P value) was used to compare survival curves of high- and low-expressing tumors.

\section{Results}

A total of 22 pediatric ependymoma cases (13 (59.1\%) males and nine $(40.9 \%)$ females) satisfied the inclusion criteria. The mean age upon diagnosis was 4.22 years (range: 1 - 9 years). Approximately two-thirds of the ependymomas were located in the infratentorial compartment $(\mathrm{n}=14,63.6 \%)$, while a third were found in the supratentorial compartment $(\mathrm{n}=8,36.3 \%)$. Five tumors were WHO grade II $(22.7 \%)$, while 17 tumors were WHO grade III (77.2\%). The median event-free survival was 1.57 years (range: $0.00-6.47$ ), while the median overall survival (OS) was 1.96 years (range: 0.01 - 7.37).

High expression of p16, EZH2, FOXJ1, and tenascin was observed in $59.0 \%, 36.3 \%, 40.9 \%$, and $68.1 \%$ of the ependymoma samples, respectively (Fig. 1). Cases with high and low p16 expression had an overall median survival time of 5.38 and 3.38 years and a cumulative OS rate of $44.5 \%$ and $36.5 \%$, respectively. Low p16 expression was significantly associated with poorer outcomes $(\mathrm{P}=0.009)$ (Fig. 2a).

The cumulative OS rates for tumors with high expression of EZH2, FOXJ1, and tenascin were 43.7\%, 51.9\%, and $42.9 \%$, respectively. No relationship was observed between EZH2, FOXJ1, and tenascin expression and OS (P = 0.904, 0.844 , and 0.646 , respectively) (Fig. $2 \mathrm{~b}-\mathrm{d}$ ). The cumulative relapse-free survival (RFS) rates for tumors with high expression of $\mathrm{p} 16, \mathrm{EZH} 2, \mathrm{FOXJ} 1$, and tenascin were $11.1 \%$, $16.7 \%, 14.3 \%$, and $9.1 \%$, respectively. No relationship was observed between p16, EZH2, FOXJ1, and tenascin expression and RFS ( $\mathrm{P}=0.900,0.373,0.411,0.166$, respectively) (Fig. 3a-d).

\section{Discussion}

Intracranial ependymomas are clinically and biologically diverse tumors whose behavior is very difficult to predict based on clinical factors $[8,9]$. Studies have shown that histopathologic WHO grading does not reliably correlate with clinical 

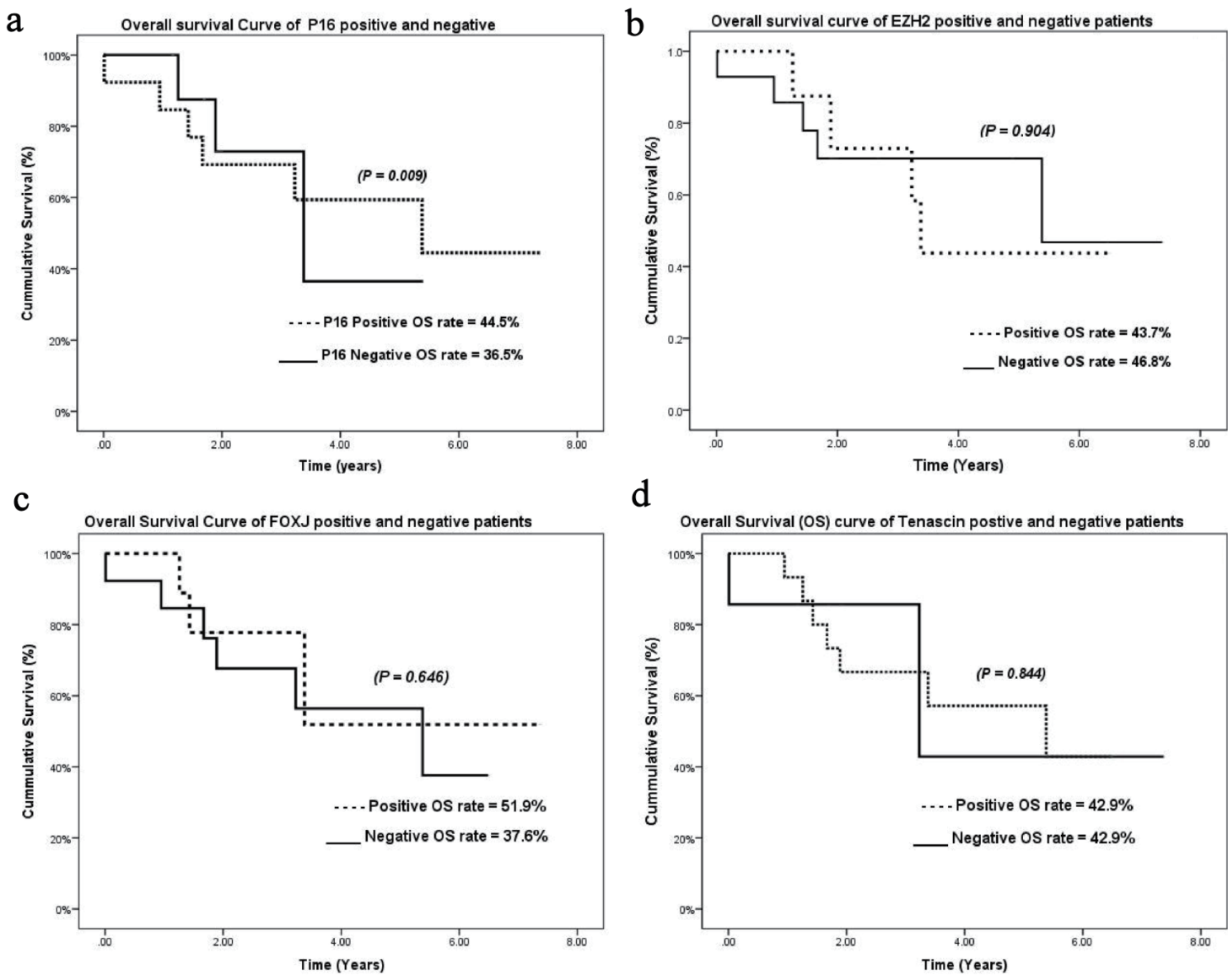

d

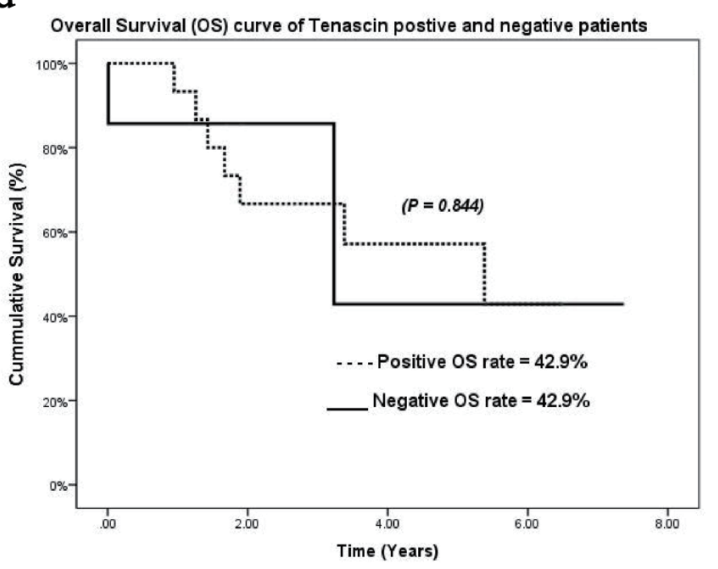

Figure 2. Kaplan-Meier overall survival curves for (a) p16, (b) EZH2, (c) FOXJ1, and (d) tenascin. EZH2: enhancer of zeste homolog 2; FOXJ1: forkhead box protein $\mathrm{J} 1$.

outcomes [8]. Thus, identifying prognostic markers for intracranial ependymoma that will stratify patients for appropriate therapy is necessary.

P16, a protein encoded by the tumor suppressor gene cyclin-dependent kinase inhibitor $2 \mathrm{~A}(C D K N 2 A)$ located on chromosome 9 , is a critical player in the cell cycle, slowing the progression from G1 phase to S phase. After staining 16 cases of intracranial ependymoma using immunohistochemistry with a p16 monoclonal antibody, Bortolotto et al found that three were negative for $\mathrm{p} 16$ immunostaining, one of which corresponded to a WHO grade III ependymoma [10].

Rousseau et al found promoter methylation for $C D K N 2 A$ in $21 \%$ of ependymal tumors, as well as lower methylation in posterior fossa tumors among children than adults [11].

In their cohort of 122 patients with ependymoma, Korshunov and his colleagues studied DNA copy-number aberrations using array-based comparative genomic hybridization. Accordingly, they found that homozygous deletion of $C D K N 2 A$ was a powerful independent indicator of unfavorable clinical outcomes [9].

Godfraind et al who examined intracranial ependymomas using interphase fluorescent in situ hybridization, found no correlation between chromosome locus 9p21 (CDKN2A) and prognosis [6].

Using a tissue microarray to study several biomarkers, Modena et al found no significant association between CDKN2A and OS and RFS after multivariate analyses [12].

$\mathrm{Li}$ et al performed immunostaining for EZH2, Ki-67, B lymphoma Moloney murine leukemia virus insertion region 1 homolog, p16, Y-box binding protein 1, phosphorylated protein kinase $\mathrm{B}$, and epidermal growth factor receptor in 180 ependymoma samples from 12 Canadian pediatric centers [8]. They found that EZH2 expression was an independent indicator of poor outcome. Likewise, they revealed that the absence of p16 expression was associated with poor prognosis among patients with supratentorial ependymoma. The present study similarly showed that low p16 expression was associated with poor prognosis. However, our study found no correlation between EZH2 expression and survival.

Abedalthagafi et al found that FOXJ1 expression was preserved in many well-differentiated neoplasms arising along the ventricle, including low-grade ependymomas with reduced expression in anaplastic ependymomas [13]. However, the present study found no relationship between FOXJ1 expression and survival.

A multicentric European study evaluated prognostic 


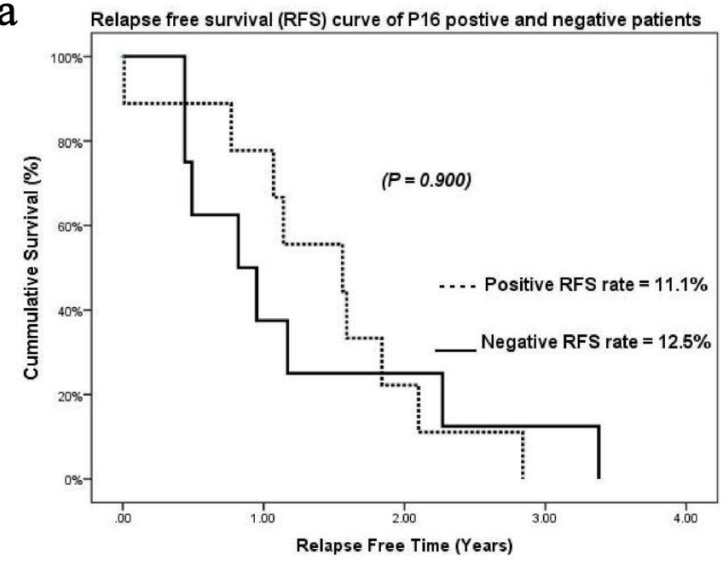

C

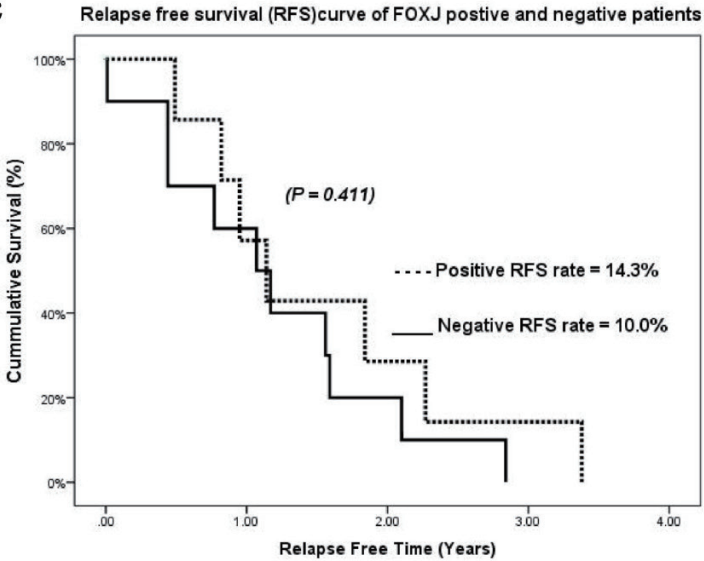

b

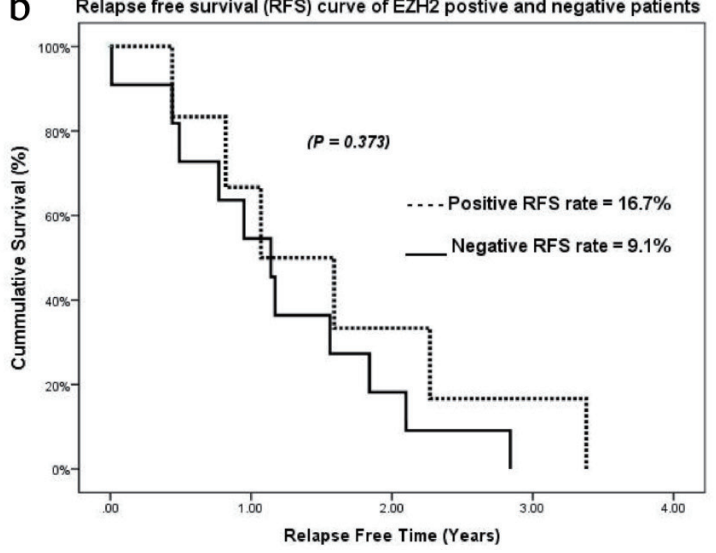

d Relapse free survival (RFS) curve of Tenascin postive and negative patients

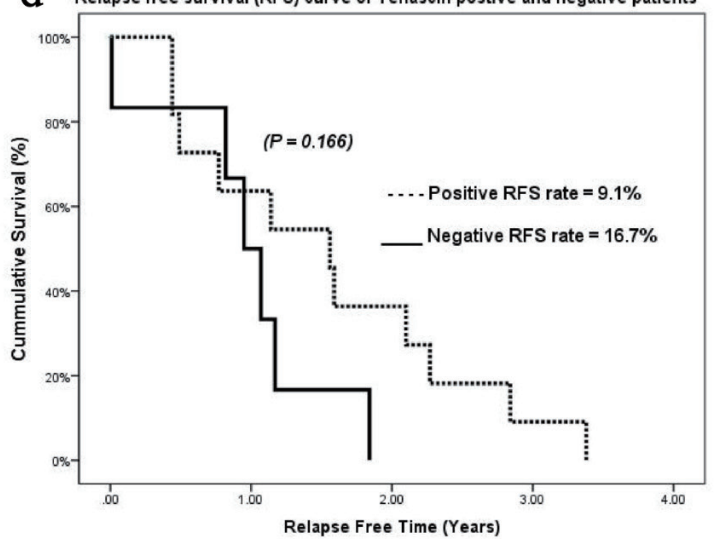

Figure 3. Kaplan-Meier relapse-free survival curves for (a) p16, (b) EZH2, (c) FOXJ1, and (d) tenascin. EZH2: enhancer of zeste homolog 2; FOXJ1: forkhead box protein $\mathrm{J} 1$.

biomarkers tenascin- $\mathrm{C}$ expression and 1q25 gain in a pooled analysis of five independent cohorts and found that tenascin- $\mathrm{C}$ and 1q25 gain were significantly associated with age at diagnosis and posterior fossa tumor location [14]. Moreover, chromosome 1q25 status added independent prognostic value for death beyond classical variables, while the prognostic utility of tenascin-C was dependent on tumor location. However, the present study found no relationship between tenascin expression and $\mathrm{OS}(\mathrm{P}=0.64)$, which could be attributed to our small sample size.

In our cohort of patients with ependymoma, loss of p16 expression was associated with inferior OS but not RFS. This could be attributed to promoter methylation [11] or homozygous deletion [9] of $C D K N 2 A$ (p16). Moreover, no correlation was observed between the remainder of the biomarkers (EZH2, FOXJ1, and tenascin) and OS or RFS.

\section{Conclusions}

Our findings showed that loss of p16 expression was associated with inferior prognosis among patients with ependymomas and may be used for risk stratification. The strength of the current research was our use of whole-tissue blocks and not microarrays for immunohistochemistry, which provides better representation of reactivity. The limitations of our study include its small sample size and variable sensitivity of different antibody clones and detection methods.

\section{Acknowledgments}

None to declare.

\section{Financial Disclosure}

This research was generously supported by a grant from Sanad Children's Cancer Support Association (grant number RGB2016-06).

\section{Conflict of Interest}

The authors declare that they have no conflict of interest. 


\section{Informed Consent}

The research utilized archival tissue blocks at the Department of Pathology, and written informed consent was exempted by the Institutional Review Board (IRB).

\section{Author Contributions}

Dr. Sadeq Al-Dandan: overall conduct of study and ethical considerations; supervision of team (research assistants, co-investigators, biostatistician and consultant); manage the financial aspects of research (equipment, consumables, and payment to coordinators, co-investigators and consultant); report the status of the study and complete IRF progress reports; review of the database and ensure correct clinical and pathological data; review histology slides in order to reach a consensus on ambiguous diagnoses with the consultant; review of the pathological parameters into the data collection sheets; choose appropriate blocks for immunohistochemical testing; analysis of the immunohistochemical data with the clinical and pathological parameters in collaboration with the biostatistician; write and review of the manuscript; submission of manuscript to journals for publication. Dr. Syed Nizam Shah: collection of clinical parameters in the data collection sheet; review of the clinical parameters in the data collection sheet; assist in analysis of the immunohistochemical data with the clinical and pathological parameters in collaboration with the biostatistician; assist in write and review of the manuscript. Dr. Musa AlHarbi: review of the clinical parameters in the data collection sheet; assist in analysis of the immunohistochemical data with the clinical and pathological parameters in collaboration with the biostatistician; assist in write and review of the manuscript.

\section{Data Availability}

The data supporting the findings of this study are available from the corresponding author upon reasonable request.

\section{References}

1. Zakrzewska M, Fendler W, Zakrzewski K, Sikorska B, Grajkowska W, Dembowska-Baginska B, Filipek I, et al. Altered MicroRNA expression is associated with tumor grade, molecular background and outcome in childhood infratentorial ependymoma. PLoS One. 2016;11(7):e0158464.

2. Taylor MD, Poppleton H, Fuller C, Su X, Liu Y, Jensen $\mathrm{P}$, Magdaleno S, et al. Radial glia cells are candidate stem cells of ependymoma. Cancer Cell. 2005;8(4):323-335.

3. Massimino M, Miceli R, Giangaspero F, Boschetti L, Modena P, Antonelli M, Ferroli P, et al. Final results of the second prospective AIEOP protocol for pediatric intracranial ependymoma. Neuro Oncol. 2016;18(10):14511460.

4. Andreiuolo F, Ferreira C, Puget S, Grill J. Current and evolving knowledge of prognostic factors for pediatric ependymomas. Future Oncol. 2013;9(2):183-191.

5. Araki A, Chocholous M, Gojo J, Dorfer C, Czech T, Heinzl H, Dieckmann K, et al. Chromosome 1q gain and tenascin- $\mathrm{C}$ expression are candidate markers to define different risk groups in pediatric posterior fossa ependymoma. Acta Neuropathol Commun. 2016;4(1):88.

6. Godfraind C, Kaczmarska JM, Kocak M, Dalton J, Wright KD, Sanford RA, Boop FA, et al. Distinct diseaserisk groups in pediatric supratentorial and posterior fossa ependymomas. Acta Neuropathol. 2012;124(2):247-257.

7. Mack SC, Witt H, Piro RM, Gu L, Zuyderduyn S, Stutz AM, Wang $X$, et al. Epigenomic alterations define lethal CIMP-positive ependymomas of infancy. Nature. 2014;506(7489):445-450.

8. Li AM, Dunham C, Tabori U, Carret AS, McNeely PD, Johnston D, Lafay-Cousin L, et al. EZH2 expression is a prognostic factor in childhood intracranial ependymoma: a Canadian Pediatric Brain Tumor Consortium study. Cancer. 2015;121(9):1499-1507.

9. Korshunov A, Witt H, Hielscher T, Benner A, Remke M, Ryzhova M, Milde T, et al. Molecular staging of intracranial ependymoma in children and adults. J Clin Oncol. 2010;28(19):3182-3190.

10. Bortolotto S, Chiado-Piat L, Cavalla P, Bosone I, Mauro A, Schiffer D. CDKN2A/p16 in ependymomas. J Neurooncol. 2001;54(1):9-13.

11. Rousseau E, Ruchoux MM, Scaravilli F, Chapon F, Vinchon M, De Smet C, Godfraind C, et al. CDKN2A, CDKN2B and p14ARF are frequently and differentially methylated in ependymal tumours. Neuropathol Appl Neurobiol. 2003;29(6):574-583.

12. Modena P, Buttarelli FR, Miceli R, Piccinin E, Baldi C, Antonelli M, Morra I, et al. Predictors of outcome in an AIEOP series of childhood ependymomas: a multifactorial analysis. Neuro Oncol. 2012;14(11):1346-1356.

13. Abedalthagafi MS, Wu MP, Merrill PH, Du Z, Woo T, Sheu SH, Hurwitz S, et al. Decreased FOXJ1 expression and its ciliogenesis programme in aggressive ependymoma and choroid plexus tumours. J Pathol. 2016;238(4):584-597.

14. Andreiuolo F, Le Teuff G, Bayar MA, Kilday JP, Pietsch T, von Bueren AO, Witt $\mathrm{H}$, et al. Integrating Tenascin-C protein expression and 1q25 copy number status in pediatric intracranial ependymoma prognostication: A new model for risk stratification. PLoS One. 2017;12(6):e0178351. 